\title{
Joint Channel-and-Network Coding Using EXIT Chart Aided Relay Activation
}

\author{
Shinsuke Ibi, Seiichi Sampei \\ Dept. of Information and Communications Technol., \\ Osaka Univesity, 2-1 Yamada-oka, Suita, 565-0871, Japan \\ Email: \{ibi, sampei\}@comm.emg.osaka-u.ac.jp
}

Abstract-This paper presents a relay activation scheme de-
signed for joint channel-and-network (JCN) coded systems rely-
ing on an iterative decoding. A primary focus is on proposing
criteria of the relay activation to find the best user combination
for cooperative relaying, which exploits extrinsic information
transfer (EXIT) chart analysis. We will demonstrate that the
EXIT chart aided relay activation scheme is capable of reduc-
ing the probability of outages, despite increasing the effective
throughput of network.
Index Terms-User cooperation, network coding, channel cod-
ing, iterative decoding, multi-hop, cooperative relaying, EXIT
chart analysis.

\section{INTRODUCTION}

User cooperation has attracted significant research attention owing to its spatial diversity benefits [1], [2]. Relaying or multi-hop transmissions constitute the simplest form of user cooperation. However, due to the creation of the broadcast and cooperation phases, they achieve a high diversity gain at the cost of a factor two multiplexing loss. To overcome this deficiency, the adoption of network coding has been studied, where we multiplex several users' information by their algebraic superposition over a finite field [3], [4]. As a benefit of the network coding, the above-mentioned half duplex multiplexing loss is mitigated without sacrificing the diversity gain. The designs of joint channel-and-network (JCN) codes and their iterative detection have been investigated in [5]-[7]. In [5], a simple triangular topology conceived for two-user cooperation was combined with beneficial channel codes. As further advances, two-way relay channels (TWRC) and multiple-access relay channels (MARC) were investigated in [6] and [7], respectively, where a turbo-like code was constituted for transmissions over the TWRC and MARC in order to achieve a high diversity gain.

The primary focus of this paper is on finding beneficial relay activation criteria for a simple triangular topology. In sophisticated JCN coded systems, extrinsic information transfer (EXIT) chart analysis may be involved for visualizing the convergence behavior of the iterative detection scheme [8], [9]. Against this background, the novel contribution of this treatise is that an EXIT-chart-aided relay activation scheme is proposed for a low-complexity XOR assisted JCN code.

The financial support of the EU under the auspices of the Optimix project and the Telecommunications Advancement Foundation, Japan is gratefully acknowledged.

\author{
Lajos Hanzo \\ School of ECS, University of Southampton, \\ 4004 Mountbatten Bdg., Salisbury Rd. SO17 1BJ, UK \\ Email:1h@ecs.soton.ac.uk
}

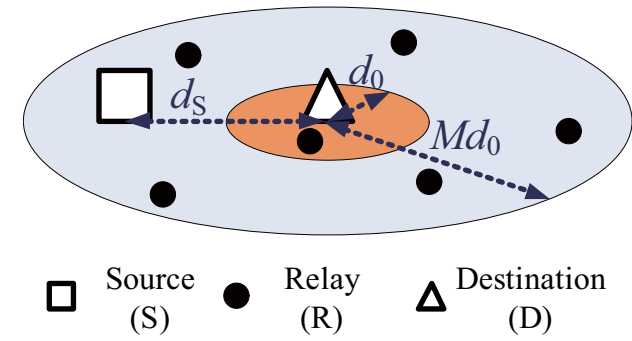

(S)

(R)

(D)

Fig. 1. Network model constituted by source (S), relay (R) and destination (D) nodes.

The rest of this paper is organized as follows. In Sect. II, the problems to be solved in this paper are clarified in the context of the network and signal models considered. Then, the iterative decoding scheme designed for the XOR assisted transmission is analyzed with the aid of EXIT chart in Sect. III. A novel EXIT chart aided relay activation scheme is proposed in Sect. IV. The scheme is characterized with the aid of computer simulations in Sect. V. Finally, the paper is concluded by a brief summary in Sect. VI.

\section{System Model and Problem Statements}

\section{A. Network model}

The network model considered is shown in Fig. 1. For the sake of simplicity, we assume here that there is a single source (S)-and-destination (D) pair separated by the distance $d_{\mathrm{S}}$ in the network. The S-D link is assumed to be subject to path loss, shadowing and quasi-static flat fading, where $d_{0}$ denotes the distance bound, which guarantees a block error rate (BLER) $\leq \omega$ over all channel realizations. Naturally, we have BLER $>\omega$ when $d_{\mathrm{S}}>d_{0}$. Furthermore, let us assume that $N_{\mathrm{R}}$ relay $(\mathrm{R})$ nodes are uniformly distributed within a circular area having a radius of $M d_{0}$.

If our objective is simply to improve the achievable BLER for $d_{\mathrm{S}}>d_{0}$, multi-hop transmissions constitute a straightforward solution [10]. However, even though the channel capacity for each link is enhanced owing to the shortened distance, the end-to-end throughput between the $\mathrm{S}$ and $\mathrm{D}$ nodes is not significantly improved, since the multiple hops require extra radio resources. In other words, multiple copies of the information message have to be relayed. Hence, our goal is to reduce the amount of extra radio resources required 
by exploiting not only two-hop aided but also XOR assisted transmissions ${ }^{1}$.

The $\mathrm{D}$ node should send a re-transmission request to an appropriate $\mathrm{R}$ node, which can perfectly detect messages from the $\mathrm{S}$ node. More specifically, if the cyclic redundancy check (CRC) of the S-D link returns an indicator of successful detection, no relay node is activated and the session is completed by "direct transmissions." Otherwise, assuming that the D node is informed of the list of potential R nodes, which have perfectly decoded the S's message, as well as assuming that the D node is aware of each channel's SNR between the potential R and $\mathrm{D}$ nodes with the aid of appropriate control channels, the D node requests re-transmission from an appropriate potential $\mathrm{R}$ node by activating "two-hop transmission" or "XOR assisted transmission." The main problem to be solved is to formulate a decision, whether to activate "two-hop transmission" or "XOR assisted transmission" in the potential R nodes distributed in Fig. 1.

\section{B. Signal model}

The signal models of the S-D and R-D links are characterized as follows. At the transmitters of the $\mathrm{S}$ and $\mathrm{R}$ nodes, the information bits to be sent to the $\mathrm{D}$ node, namely the variables $c_{i}(l), i \in\{\mathrm{S}, \mathrm{R}\}$ are mapped to the transmitted symbols $s_{i}(k)$ each representing one of the $Q$ constellation points $\mathcal{S} \in\left\{\mathcal{S}_{0}, \ldots, \mathcal{S}_{Q-1}\right\}$.

Let the joint impact of path loss, shadowing and quasistatic flat fading be denoted by the coefficient $h_{i}$. The received symbol observed at the receiver of the D node is expressed as

$$
r_{i}(k)=h_{i} s_{i}(k)+n_{i}(k),
$$

where $k$ represents the discrete time index, $n_{i}(k)$ is the complex-valued white Gaussian noise process of $\mathcal{C N}\left(0, N_{0}\right)$ and $N_{0}$ denotes the one-sided noise power spectral density.

The symbol-to-bit demapper of the $\mathrm{D}$ node calculates the extrinsic log likelihood ratio (LLR) $\alpha_{i}(l)$, which is defined by

$$
\alpha_{i}(l)=\ln \frac{\operatorname{Pr}\left[r_{i}(k) \mid c_{i}(l)=1\right]}{\operatorname{Pr}\left[r_{i}(k) \mid c_{i}(l)=0\right]} .
$$

For the ease of analysis, in this paper, we assume the adoption of Gray coded QPSK, having the phases of $\mathcal{S}=\{(-1-$ $\sqrt{-1}) w,(-1+\sqrt{-1}) w,(1-\sqrt{-1}) w,(1+\sqrt{-1}) w\}$ where $w=\sqrt{E_{s} / 2}$ and $E_{s}$ is the energy of each transmitted symbol. The Gray QPSK mapping allows us to rewrite (2) as

$$
\begin{array}{r}
\alpha_{i}(l=2 k-1)=\frac{2 \sqrt{2 E_{s}}}{N_{0}} \Re\left[h_{i}^{*} r_{i}(k)\right], \\
\alpha_{i}(l=2 k)=\frac{2 \sqrt{2 E_{s}}}{N_{0}} \Im\left[h_{i}^{*} r_{i}(k)\right],
\end{array}
$$

where $\Re[\cdot]$ and $\Im[\cdot]$ denote real and imaginary parts of complex values, respectively. Substituting (1) into (3) and (4), the extrinsic LLRs $\alpha_{i}(l)$ may be regarded as the received constellation points of BPSK signalling over Gaussian channels,

\footnotetext{
${ }^{1}$ In this contribution, we focus on two-hop transmissions, since the employment of multiple $\mathrm{R}$ nodes may reduce effective throughput, as mentioned in [11].
}

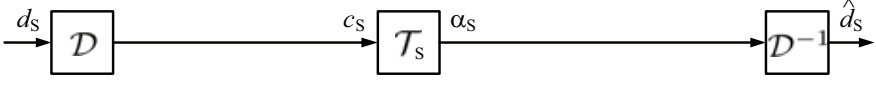

(a) Direct transmissions

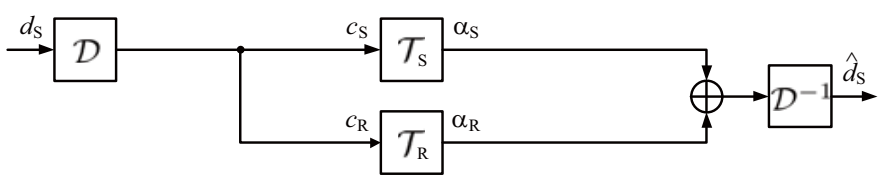

(b) Two-hop transmissions

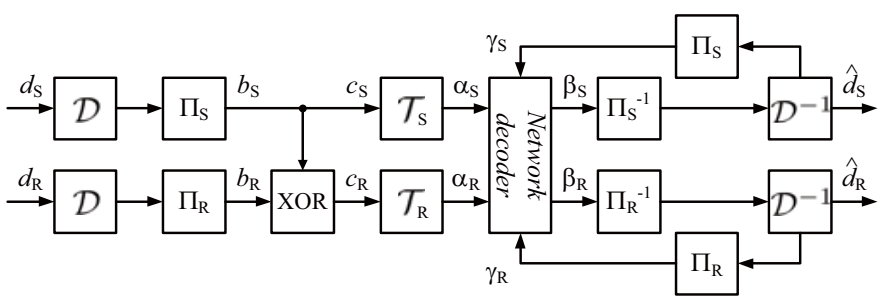

(c) XOR assisted transmissions

Fig. 2. Schematics of the three transceiver modes, where $\mathcal{D}$ and $\mathcal{D}^{-1}$ denote the channel encoder and decoder, respectively. Furthermore, $\Pi_{i}$ and $\Pi_{i}^{-1}$ are the bit interleaver and deinterleaver, respectively while $\mathcal{T}_{i}$ indicates the Gaussian LLR signal model $\mathcal{T}\left(\sigma_{i}^{2}\right)$ defined in (5).

as defined by

$$
\alpha_{i}(l)=\mu_{i} \tilde{c}_{i}(l)+\nu_{i}(l) \triangleq \mathcal{T}\left(\sigma_{i}^{2}\right),
$$

where we have $\tilde{c}_{i}(l)=2 c_{i}(l)-1$ and $\nu_{i}(l)$ represents the zeromean real-valued white Gaussian noise process of $\mathcal{N}\left(0, \sigma_{i}^{2}\right)$. The channel gain $\mu_{i}$ and the variance $\sigma_{i}^{2}$ are given by $\sigma_{i}^{2}=4 \eta_{i}$ and $\mu_{i}=\frac{\sigma_{i}^{2}}{2}$, where $\eta_{i}$ denotes the instantaneous received SNR, which is defined by $\eta_{i}=\left|h_{i}\right|^{2} \frac{E_{s}}{N_{0}}$.

Since the ratio of the channel gain $\mu_{i}$ and of the variance $\sigma_{i}^{2}$ obeys $\mu_{i}=\sigma_{i}^{2} / 2$, the consistency condition of the probability density function for $\alpha_{i}(l)$ is satisfied [12]. Therefore, the mutual information (MI) of (5), which is equivalent to the channel capacity constrained by BPSK signaling, may be closely-approximated by

$$
I_{i}=J\left(\sigma_{i}\right)=J\left(2 \sqrt{\eta_{i}}\right) \approx\left(1-2^{-H_{1} \sigma_{i}^{2 H_{2}}}\right)^{H_{3}},
$$

where the mapping-specific parameters are $H_{1}=0.3073$, $H_{2}=0.8935$, and $H_{3}=1.1064$, which are obtained by leastsquared curve fitting [13]. Furthermore, the inverse function of (6) is given by

$$
\sigma_{i}=J^{-1}\left(I_{i}\right) \approx\left(-\frac{1}{H_{1}} \log _{2}\left(1-I_{i}^{\frac{1}{H_{3}}}\right)\right)^{\frac{1}{2 H_{2}}} .
$$

\section{Transciever schematic for each transmission mode}

The transceiver schematics designed for the three transmission modes considered are depicted in Fig. 2.

1) Direct transmissions: As shown in Fig. 2(a), the information bits $d_{\mathrm{S}}(m)$ to be sent to the $\mathrm{D}$ node from the $\mathrm{S}$ node transmitter, are encoded by a channel encoder in order to form the coded bits $c_{\mathrm{S}}(l)$. The coded bits $c_{\mathrm{S}}(l)$ are mapped to the transmitted symbols, sent over the wireless channel and demapped to generate the extrinsic LLRs $\alpha_{\mathrm{S}}(l)$. The 
extrinsic LLRs $\alpha_{\mathrm{S}}(l)$ are delivered to the channel decoder (CD), which outputs the estimated information bits $\hat{d}_{\mathrm{S}}(m)$ after error correction decoding.

2) Two-hop transmissions: Recall that the appropriately selected $\mathrm{R}$ node has perfect knowledge of the information message $d_{\mathrm{S}}(m)$, as shown in Fig. 2(b). Once the D node's CRC detects an error, a copy of the message is relayed via the $\mathrm{R}$ node to the $\mathrm{D}$ node. At the receiver, the extrinsic LLRs $\alpha_{\mathrm{S}}(l)$ provided by the $\mathrm{S}$ node assist us in detecting the information message $d_{\mathrm{S}}(m)$ of Fig. 2(b). Since the LLRs $\alpha_{\mathrm{S}}(l)$ and $\alpha_{\mathrm{R}}(l)$ are conveyed over independent channels during the first broadcast and the second cooperative phases, respectively, the extrinsic LLRs of the joint probability are given by

$\begin{aligned} \ln \frac{\operatorname{Pr}\left[r_{\mathrm{S}}(k), r_{\mathrm{R}}(k) \mid c_{i}(l)=1\right]}{\operatorname{Pr}\left[r_{\mathrm{R}}(k), r_{\mathrm{R}}(k) \mid c_{i}(l)=0\right]} & =\ln \prod_{i} \frac{\operatorname{Pr}\left[r_{i}(k) \mid c_{i}(l)=1\right]}{\operatorname{Pr}\left[r_{i}(k) \mid c_{i}(l)=0\right]} \\ & =\alpha_{\mathrm{S}}(l)+\alpha_{\mathrm{R}}(l) .\end{aligned}$

Instead of simply using $\alpha_{\mathrm{S}}(l)$ as in conventional direct transmission, the combined LLRs are delivered to the $\mathrm{CD}$, in order to derive more reliable estimates of the information bits $\hat{d}_{\mathrm{S}}(m)$.

3) XOR assisted transmissions: Since the selected R node has its own message for transmission to the $\mathrm{D}$ node in this mode, each information message $d_{i}(m)$ is independently encoded and interleaved, in order to generate the coded bits $b_{i}(l)$. Then, the XORed bits of $c_{\mathrm{R}}(l)=b_{\mathrm{S}}(l) \oplus b_{\mathrm{R}}(l)$ are generated, where the null element in $\mathrm{GF}(2)$ is assumed to be zero. The XORed bits $c_{\mathrm{R}}(l)$ are then mapped to the QPSK symbols, transmitted over the wireless channels, and then the extrinsic LLRs $\alpha_{\mathrm{R}}(l)$ are generated.

If we focus our attention purely on $\alpha_{\mathrm{R}}(l)$, we encounter the following ambiguity problem. For example, it is impossible to judge, whether $b_{\mathrm{S}}=b_{\mathrm{R}}=1$ or $b_{\mathrm{S}}=b_{\mathrm{R}}=0$ is correct, when we have $c_{R}=0$, since the XORed value of $c_{R}$ becomes zero for both cases. In order to resolve this ambiguity problem, the pre-calculated extrinsic LLR $\alpha_{S}(l)$ assists in detecting the information message $d_{\mathrm{S}}(m)$ and $d_{\mathrm{R}}(m)$ with the aid of the iterative detection process of Fig. 2(c), where different random interleavers are employed, in order to improve the attainable performance.

Maximum a-posteriori probability (MAP) detection is applied in order to decompose the XORed bits of $c_{\mathrm{S}}(l)$ and $c_{\mathrm{R}}(l)$ into the corresponding pair of coded bits $b_{\mathrm{S}}(l)$ and $b_{\mathrm{R}}(l)$. We refer to this process as the "Network decoder (ND)." The ND provides the extrinsic LLRs $\beta_{i}(l)$ for the coded bits $b_{i}(l)$. After de-interleaving the LLRs $\beta_{i}(l)$, they are delivered to the CDs of Fig. 2(c), in order to calculate the a-priori LLRs $\gamma_{i}(l)$, which are forwarded to the ND via the interleavers $\Pi_{S}$ and $\Pi_{\mathrm{R}}$ as a-priori probabilities. The exchange of the LLRs $\beta_{i}(l)$ and $\gamma_{i}(l)$ between the ND and CDs is capable of resolving the above-mentioned ambiguity problem. After several iterations, the estimated information bits $\hat{d}_{\mathrm{S}}(m)$ and $\hat{d}_{\mathrm{R}}(m)$ are obtained by the CDs.

A specific problem to be considered here is that the ND process cannot always resolve the XORed bits, since their separability depends on the SNRs experienced in both the S-D and R-D links. Hence, this relationship is investigated in the next section.

\section{ITERATIVE Detection of XOR Assisted Relaying}

Without loss of generality, the indices $m, l$ and $k$ are omitted for ease of notational simplicity.

\section{A. Iterative decoding of the JCN codes}

After observing $\alpha_{\mathrm{S}}$ and $\alpha_{\mathrm{R}}$, the extrinsic LLRs $\beta_{i}$ are derived by the ND, expressed as

$$
\beta_{i}=\ln \frac{\operatorname{Pr}\left[b_{i}=1 \mid \alpha_{\mathrm{S}}, \alpha_{\mathrm{R}}\right]}{\operatorname{Pr}\left[b_{i}=0 \mid \alpha_{\mathrm{S}}, \alpha_{\mathrm{R}}\right]}-\gamma_{i},
$$

where $\gamma_{i}$ is an a-priori LLR fed back from each CD to the $\mathrm{ND}$, as defined by $\gamma_{i}=\ln \frac{\operatorname{Pr}\left[b_{i}=1\right]}{\operatorname{Pr}\left[b_{i}=0\right]}$. Note that $\gamma_{i}$ is zero at the first iteration, because no $a$-priori knowledge is available about $b_{i}$ at this stage. In order to derive the a-posteriori probability $\operatorname{Pr}\left[b_{i} \mid \alpha_{\mathrm{S}}, \alpha_{\mathrm{R}}\right]$ in (9), the marginalization of the joint probability and Bayes' theorem play a key role, yielding

$$
\begin{aligned}
\operatorname{Pr}\left[b_{i} \mid \alpha_{\mathrm{S}}, \alpha_{\mathrm{R}}\right] & =\sum_{b_{j}} \operatorname{Pr}\left[b_{\mathrm{S}}, b_{\mathrm{R}} \mid \alpha_{\mathrm{S}}, \alpha_{\mathrm{R}}\right] \\
& \propto \sum_{b_{j}} \operatorname{Pr}\left[\alpha_{\mathrm{S}}, \alpha_{\mathrm{R}} \mid b_{\mathrm{S}}, b_{\mathrm{R}}\right] \operatorname{Pr}\left[b_{\mathrm{S}}, b_{\mathrm{R}}\right],(10)
\end{aligned}
$$

where we have $j \neq i(i, j \in\{\mathrm{S}, \mathrm{R}\})$. Since the S-D and R-D links experience independent fading during the two different transmission phases, the joint probability may be decomposed to

$$
\begin{aligned}
& \operatorname{Pr}\left[\alpha_{\mathrm{S}}, \alpha_{\mathrm{R}} \mid b_{\mathrm{S}}, b_{\mathrm{R}}\right]=\operatorname{Pr}\left[\alpha_{\mathrm{S}} \mid b_{\mathrm{S}}, b_{\mathrm{R}}\right] \operatorname{Pr}\left[\alpha_{\mathrm{R}} \mid b_{\mathrm{S}}, b_{\mathrm{R}}\right] \\
& =\operatorname{Pr}\left[\alpha_{\mathrm{S}} \mid c_{\mathrm{S}}\right] \operatorname{Pr}\left[\alpha_{\mathrm{R}} \mid c_{\mathrm{R}}=b_{\mathrm{S}} \oplus b_{\mathrm{R}}\right] .
\end{aligned}
$$

Furthermore, since the bits $b_{\mathrm{S}}$ and $b_{\mathrm{R}}$ are generated from independent random sources, we have $\operatorname{Pr}\left[b_{\mathrm{S}}, b_{\mathrm{R}}\right]=\operatorname{Pr}\left[b_{\mathrm{S}}\right] \operatorname{Pr}\left[b_{\mathrm{R}}\right]$.

Let $\mathcal{C}_{i}$ and $\mathcal{B}_{i}$ be the ratios of the LLR $\alpha_{i}$ and of the coded bit $b_{i}$ defined by

$$
\mathcal{C}_{i}=\frac{\operatorname{Pr}\left[\alpha_{i} \mid c_{i}=1\right]}{\operatorname{Pr}\left[\alpha_{i} \mid c_{i}=0\right]}, \quad \mathcal{B}_{i}=\frac{\operatorname{Pr}\left[b_{i}=1\right]}{\operatorname{Pr}\left[b_{i}=0\right]} .
$$

After some simple algebraic manipulations, (9) is rewritten as

$$
\begin{aligned}
& \beta_{\mathrm{S}}=\ln \mathcal{C}_{\mathrm{S}}+\ln \frac{\mathcal{C}_{\mathrm{R}}+\mathcal{B}_{\mathrm{R}}}{1+\mathcal{C}_{\mathrm{R}} \mathcal{B}_{\mathrm{R}}}, \\
& \beta_{\mathrm{R}}=\ln \frac{\mathcal{C}_{\mathrm{R}}+\mathcal{C}_{\mathrm{S}} \mathcal{B}_{\mathrm{S}}}{1+\mathcal{C}_{\mathrm{R}} \mathcal{C}_{\mathrm{S}} \mathcal{B}_{\mathrm{S}}} .
\end{aligned}
$$

By exploiting that the extrinsic LLRs $\alpha_{i}$ may be regarded as being Gaussian distributed in conjunction with BPSK signaling, as mentioned in Sect. II.B, the ratios $\mathcal{C}_{i}$ are reduced to

$$
\mathcal{C}_{i}=\frac{\exp \left[-\left(\alpha_{i}-\frac{\sigma_{i}^{2}}{2}\right)^{2} /\left(2 \sigma_{i}^{2}\right)\right]}{\exp \left[-\left(\alpha_{i}+\frac{\sigma_{i}^{2}}{2}\right)^{2} /\left(2 \sigma_{i}^{2}\right)\right]}=\exp \left[\alpha_{i}\right] .
$$

Furthermore, since we have $\mathcal{B}_{i}=\exp \left(\gamma_{i}\right)$, (13) and (14) may be rewritten as

$$
\begin{aligned}
& \beta_{\mathrm{S}}=\alpha_{\mathrm{S}}+\left(\alpha_{\mathrm{R}} \boxplus \gamma_{\mathrm{R}}\right), \\
& \beta_{\mathrm{R}}=\alpha_{\mathrm{R}} \boxplus\left(\alpha_{\mathrm{S}}+\gamma_{\mathrm{S}}\right),
\end{aligned}
$$




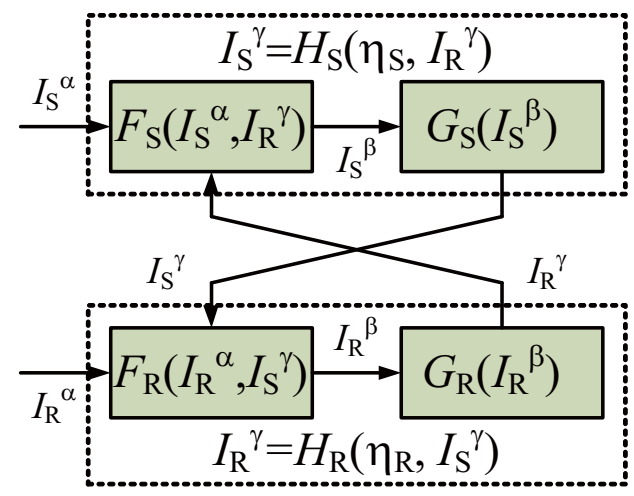

Fig. 3. EXIT model of XOR assisted transmission.

where $\boxplus$ denotes the boxplus operator defined by Hagenauer et al. [14] as ${ }^{2}$

$$
A \boxplus B=\ln \frac{\exp (A)+\exp (B)}{1+\exp (A+B)} .
$$

\section{B. EXIT chart analysis}

Figure 3 shows on EXIT chart analysis model. Based on (6) representing the variance of the LLR-to-MI conversion, the LLRs $\alpha_{i}, \beta_{i}$ and $\gamma_{i}$ are transformed to the corresponding MI, which are denoted by $I_{i}^{\alpha}, I_{i}^{\beta}$ and $I_{i}^{\gamma}$, respectively. Then, the EXIT functions, which are defined as the input/output relationships, for ND $F_{i}$ and for the $\mathrm{CD} G_{i}$ are denoted by

$$
\begin{aligned}
& I_{i}^{\beta}=F_{i}\left(I_{i}^{\alpha}, I_{j}^{\gamma}\right), \\
& I_{i}^{\gamma}=G_{i}\left(I_{i}^{\beta}\right),
\end{aligned}
$$

where we have $i \neq j$.

Recall that the function $F_{i}$ is constituted by the sum and boxplus operations, when genarating the extrinsic LLRs. According to [9], the MI after the sum and boxplus operations between the extrinsic LLRs $A$ and $B$ are derived by

$$
\begin{aligned}
& A+B \rightarrow J\left(\sqrt{\left[J^{-1}\left(I_{A}\right)\right]^{2}+\left[J^{-1}\left(I_{B}\right)\right]^{2}}\right), \\
& A \boxplus B \rightarrow 1-J\left(\sqrt{\left[J^{-1}\left(1-I_{A}\right)\right]^{2}+\left[J^{-1}\left(1-I_{B}\right)\right]^{2}}\right),
\end{aligned}
$$

where $I_{A}$ and $I_{B}$ represent the MI for the LLRs $A$ and $B$, respectively ${ }^{3}$. Substituting these MI calculations into (16) and (17), $I_{\mathrm{S}}^{\beta}$ and $I_{\mathrm{R}}^{\beta}$ are expressed in (23) and (24).

On the other hand, the EXIT functions of the CD are uniquely determined by the channel code to be used. When a recursive systematic convolutional (RSC) encoder having the octal generator polynomials of $[1,15 / 13]_{\text {oct }}$ is employed for both the $\mathrm{S}$ and $\mathrm{R}$ nodes, the EXIT function may be approximated by

$$
I_{i}^{\gamma}=G_{i}\left(I_{i}^{\beta}\right) \approx\left(1-2^{-D_{1}\left(I_{i}^{\beta}\right)^{D_{2}}}\right)^{D_{3}},
$$

where the mapping-specific parameters are $D_{1}=17.7954$, $D_{2}=3.4600$, and $D_{3}=1.7490$, which were calculated

\footnotetext{
${ }^{2}$ In [14], the null element in $\mathrm{GF}(2)$ is +1 , while it was set to zero in this paper. Therefore, the definition of the boxplus operater has the opposite sign in comparison to [14].

${ }^{3}$ Equation (22) was originally developed for binary erasure channels, but it remains fairly accurate also for AWGN channels.
}

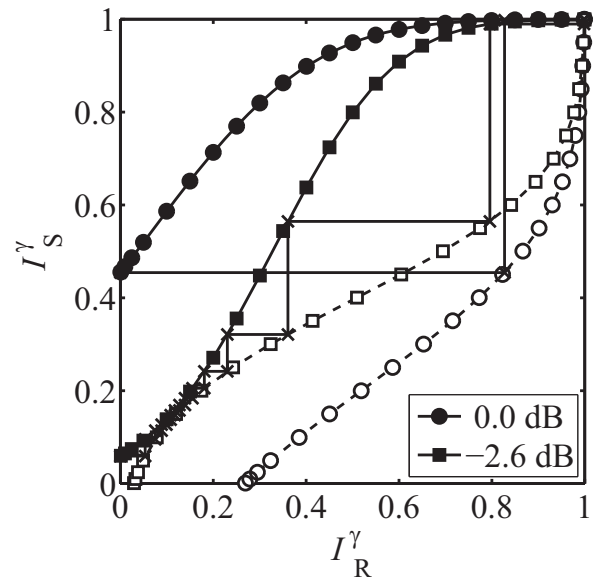

Fig. 4. EXIT chart for the XOR assisted transmission at $\mathrm{SNR} \eta_{\mathrm{R}}=5.0 \mathrm{~dB}$. The $\mathrm{dB}$ values in the legend denotes instantaneous received SNR of the S-D link $\eta_{S}$. Solid and dashed curves with black and white marker faces represent $H_{\mathrm{S}}$ and $H_{\mathrm{R}}$, respectively. Stair lines denote EXIT trajectories.

by least-squared curve fitting under the assumption of having sufficiently high code length.

Substituting (19) into (20), it is clear that $I_{i}^{\gamma}$ is uniquely determined by $I_{i}^{\alpha}$ and $I_{j}^{\gamma}$, which is expressed as

$$
I_{i}^{\gamma}=G_{i}\left[F_{i}\left(I_{i}^{\alpha}, I_{j}^{\gamma}\right)\right] \triangleq H_{i}\left(\eta_{i}, I_{j}^{\gamma}\right) .
$$

The transfer function $H_{i}$ indicates the fact that the decoder's iterative behavior may be readily characterized by observing the MI exchanges of $I_{\mathrm{S}}^{\gamma}$ and $I_{\mathrm{R}}^{\gamma}$ for a given combination of SNRs $\eta_{\mathrm{S}}$ and $\eta_{\mathrm{R}}$ without any consideration of $I_{i}^{\beta}$.

The resultant EXIT chart is depicted in Fig. 4. The instantaneous received SNR of the R-D link is fixed to $\eta_{\mathrm{R}}$ $=5.0 \mathrm{~dB}$, while that of the S-D link to $\eta_{\mathrm{S}}=0.0$ and $2.6 \mathrm{~dB}$. Figure 4 implies that the EXIT trajectories cannot reach the $(1,1)$ point for any combinations of $\eta_{\mathrm{S}}<-2.6 \mathrm{~dB}$ and $\eta_{\mathrm{R}}=5.0 \mathrm{~dB}$, since the EXIT curves intersect each other, hence the system results in detection errors. In other words, the $\mathrm{R}$ node is capable of operating without block detection error events, when $\eta_{\mathrm{S}} \geq-2.6 \mathrm{~dB}$ and $\eta_{\mathrm{R}}=5.0 \mathrm{~dB}$. However, we have to mention that there is a mismatch between decoder's convergence threshold estimated from the EXIT chart and that found by BLER simulations. This is because a sufficiently long channel code and an infinite number of iterations are assumed in the EXIT analysis, whereas an insufficiently long channel code and a finite number of iterations are assumed in the BLER simulations.

\section{EXIT Chart Aided Relay Activation}

The problem of finding an appropriate $\mathrm{R}$ node is equivalent to finding a node in the network, which satisfies the SNR requirement $\eta_{\mathrm{R}}$ under a given condition of $\eta_{\mathrm{S}}$. The most straight-forward way of finding the appropriate $\mathrm{R}$ node is to draw the EXIT trajectories for all potential $\mathrm{R}$ nodes. If the trajectory reaches the $I_{\mathrm{S}}^{\gamma}=I_{\mathrm{R}}^{\gamma} \approx 1.0$ point, the node is capable of supporting XOR assisted transmission. However, this procedure is imperfect, since there is a mismatch between the SNR requirement estimated by EXIT chart analysis and 


$$
\begin{aligned}
I_{\mathrm{S}}^{\beta} & =J\left(\sqrt{\left[J^{-1}\left(I_{\mathrm{S}}^{\alpha}\right)\right]^{2}+\left[J^{-1}\left(1-J\left(\sqrt{\left[J^{-1}\left(1-I_{\mathrm{R}}^{\alpha}\right)\right]^{2}+\left[J^{-1}\left(1-I_{\mathrm{R}}^{\gamma}\right)\right]^{2}}\right)\right)\right]^{2}}\right) \\
I_{\mathrm{R}}^{\beta} & =1-J\left(\sqrt{\left[J^{-1}\left(1-I_{\mathrm{R}}^{\alpha}\right)\right]^{2}+\left[J^{-1}\left(1-J\left(\left[J^{-1}\left(I_{\mathrm{S}}^{\alpha}\right)\right]^{2}+\left[J^{-1}\left(I_{\mathrm{S}}^{\gamma}\right)\right]^{2}\right)\right)\right]^{2}}\right) .
\end{aligned}
$$

BLER simulations. Nevertheless, it is possible to set a safetymargin in the EXIT chart based activation criterion. However, the probability of block error events caused by the mismatch is low. Therefore, the introduction of safety-margin may not be necessary.

With the objective of improving the achievable throughput of the network, we propose simple criteria for the relay activation in this section. When the CRC indicates that the direct transmission failed, the procedure refers to the list of potential $\mathrm{R}$ nodes and selects the transmission mode by obeying the following two criteria.

\section{A. Criterion for XOR assisted transmissions}

The following process is activated for each potential $\mathrm{R}$ node having own messages to be sent to the $\mathrm{D}$ node. If there are no such R nodes, we proceed to the next criterion for the two-hop transmission mode.

1) Initialize: $I_{\mathrm{S}}^{\gamma}=I_{\mathrm{R}}^{\gamma}=0.0$.

2) Update $I_{\mathrm{S}}^{\gamma}$ and $I_{\mathrm{R}}^{\gamma}$ as many times as the affordable number of iterations with the aid of the following expressions:

$$
I_{\mathrm{S}}^{\gamma}=H_{\mathrm{S}}\left(\eta_{\mathrm{S}}, I_{\mathrm{R}}^{\gamma}\right), \quad I_{\mathrm{R}}^{\gamma}=H_{\mathrm{R}}\left(\eta_{\mathrm{R}}, I_{\mathrm{S}}^{\gamma}\right) .
$$

3) Check whether both $I_{\mathrm{S}}^{\gamma}$ and $I_{\mathrm{R}}^{\gamma}$ are higher than or equal to a threshold value $I_{\mathrm{th}}$ :

$$
\left(I_{\mathrm{S}}^{\gamma} \geq I_{\mathrm{th}}\right) \quad \text { AND } \quad\left(I_{\mathrm{R}}^{\gamma} \geq I_{\mathrm{th}}\right),
$$

where $I_{\text {th }}$ is a system parameter introduced for controlling the BLER. Among the potential $\mathrm{R}$ nodes, which satisfy the above conditions, the specific node having the highest SNR $\eta_{\mathrm{R}}$ is activated for XOR assisted transmissions. If there are no such nodes, go to the next.

\section{B. Criterion for two-hop transmissions}

The following process is evaluated for each potential $\mathrm{R}$ node. If there are no such nodes, the transmissions are terminated.

1) Check wheter $\left(\eta_{\mathrm{S}}+\eta_{\mathrm{R}}\right)$ is higher than or equal to a threshold value $\eta_{\mathrm{th}}$ :

$$
\left(\eta_{\mathrm{S}}+\eta_{\mathrm{R}}\right) \geq \eta_{\mathrm{th}}
$$

where $\eta_{\mathrm{th}}$ is also a system parameter used for controlling BLER. Among the potential R nodes, which satisfy the above condition, the specific node having the highest SNR $\eta_{\mathrm{R}}$ is activated for the two-hop transmissions.

\section{Numerical Results}

In order to characterize the proposed relay activation scheme, computer simulations have been conducted. In the

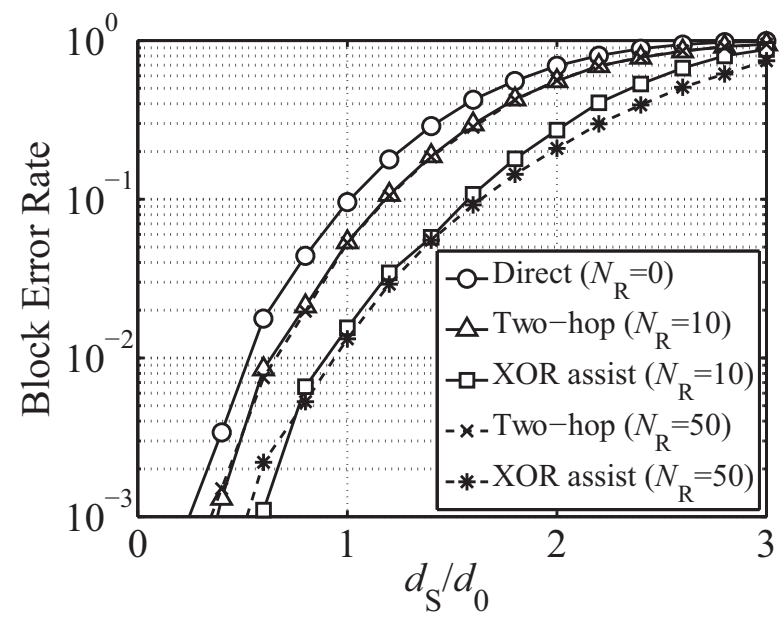

(a) Block error rate.

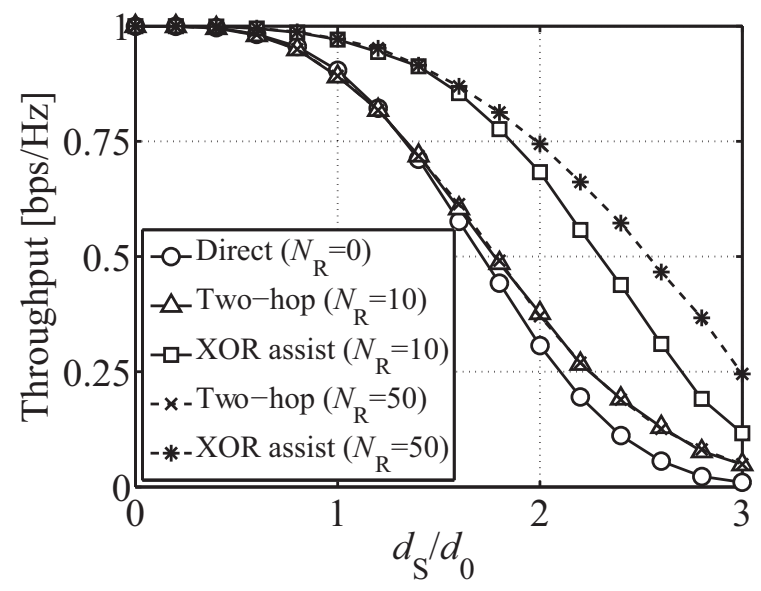

(b) Troughput.

Fig. 5. Performance of the EXIT chart aided relay activation. The dashed and solid curves show the attainable performances in the case of $N_{\mathrm{R}}=10$ and 50 , respectively.

network model of Fig. 1, we assumed having $N_{\mathrm{R}}=0,10$, or 50. Note that $N_{\mathrm{R}}=0$ is equivalent to the direct transmissions. The $\mathrm{R}$ nodes were uniformly distributed in a circular area having a radius of $3 d_{0}(M=3)$. The distance $d_{0}$ was set to a value, which satisfies BLER $\leq \omega=0.1$. For simplicity, each $\mathrm{R}$ node was assumed to always have messages ready to be sent to the $\mathrm{D}$ node in its buffer.

The path-loss exponent in the wireless network was assumed to be 3.5. Furthermore, log-normal shadowing with a standard deviation of $8 \mathrm{~dB}$ and flat Rayleigh fading having complexvalued channel coefficients obeying $\mathcal{C N}(0,1)$ were assumed. Gray coded QPSK was used for each link. In the channel 
encoders of both links, half-rate RSC schemes having a code memory of three and the octal generator polynomials of $[1,15 / 13]_{\text {oct }}$ were used. Each RSC encoder processed 1024 information bits in order to form 2048 coded bits per transmission block. The coded blocks were decoded by the Max-LogMap decoder with the Jacobian logarithm. Then, the maximum number of iterations was set to 16 . The threshold values of $I_{\mathrm{th}}$ and $\eta_{\mathrm{th}}$ were set to 0.999 and $5.21 \mathrm{~dB}$, respectively ${ }^{4}$. Information of the instantaneous received SNRs $\eta_{\mathrm{S}}$ and $\eta_{\mathrm{R}}$ were assumed to be perfectly known at the D node.

The achievable BLER and throughput for information blocks $d_{\mathrm{S}}$ are characterized in Figs. 5(a) and 5(b), respectively. The curves plotted for "Two-hop" represent transmissions without the criterion $A$ for XOR assisted transmissions. In other words, the D node evaluates only criterion B for deciding the activation of two-hop transmissions. The dashed and solid curves represent the performances for the case of $N_{\mathrm{R}}=10$ and 50 , respectively. The throughput $T$ is defined by

$$
T=\frac{(1-B L E R) N_{\mathrm{d}}}{N_{\mathrm{s}} N_{\text {phase }}} \quad[\mathrm{bps} / \mathrm{Hz}],
$$

where $N_{\mathrm{d}}$ and $N_{\mathrm{s}}$ represent the numbers of information data bits and symbols embedded in one block, respectively. $N_{\text {phase }}$ is the number of phases to be occupied for each transmission mode. Explicitly, when two-hop transmission is selected, we have $N_{\text {phase }}=2$, otherwise $N_{\text {phase }}=1$.

The horizontal axis $d_{s} / d_{0}$ denotes the distance between $\mathrm{S}$ and $\mathrm{D}$ nodes normalized by $d_{0}$. In this case, the average received SNR at the arbitrary distance $d_{i}$ is expressed as

$$
\Gamma\left(d_{i}\right)=\Gamma\left(d_{0}\right)\left(\frac{d_{i}}{d_{0}}\right)^{-3.5} \quad(i \in\{\mathrm{S}, \mathrm{R}\}),
$$

where $d_{\mathrm{R}}$ is the distance between R and D nodes, while $\Gamma\left(d_{0}\right)$ is the $\mathrm{SNR}$ required to satisfy $\mathrm{BLER}=0.1$ under the given shadowing and fading conditions. In the present simulation conditions, $\Gamma\left(d_{0}\right)$ is $25.4 \mathrm{~dB}^{5}$.

Let us now focus our attention on Fig. 5(a). Compared to direct transmissions, two-hop transmissions using $N_{\mathrm{R}}=10$ and 50 guarantees $20 \%$ extension of the cell-radius, while achieving $\mathrm{BLER}=10^{-1}$. From a different perspective, the extension implies that a $2.7 \mathrm{~dB}\left[10 \log _{10}(1.0 / 1.2)^{-3.5}\right]$ power gain can be obtained, when the distance $d_{\mathrm{S}}$ is the same for both direct and two-hop transmissions. On the other hand, XOR-assisted transmissions for $N_{\mathrm{R}}=10$ and 50 guarantee an approximately $60 \%$ extension of the coverage distance, while achieving $\mathrm{BLER}=10^{-1}$. The extension implies that a $7.1 \mathrm{~dB}$ $\left[10 \log _{10}(1.0 / 1.6)^{-3.5}\right]$ power gain can be obtained. Therefore, the EXIT chart aided relay activation is also effective in terms of reducing the required transmit power.

Next, let us focus our discussions on Fig. 5(b). Twohop transmissions cannot guarantee an increased throughput, since the extra copies of the messages occupy extra wireless resources. On the other hand, XOR-assisted transmissions

\footnotetext{
${ }^{4}$ The value of $\eta_{\mathrm{th}}=5.21 \mathrm{~dB}$ achieves BLER $=10^{-2}$ in AWGN channels.

${ }^{5}$ In order to reduce $\Gamma\left(d_{0}\right)$, space-, frequency- and time-diversity may be used. For the sake of simplicity, the diversity order was fixed to one.
}

significantly improve the attainable throughput. More specifically, at $d_{\mathrm{S}} / d_{0}=1.6$ which is the maximum distance, where we are able to maintain $\mathrm{BLER}=10^{-1}$ for the XOR assisted transmission, we can confirm a 55\% increase of the throughput compared to classic direct transmissions. Therefore, it is clear that XOR-assisted transmissions are capable of simultaneously improving both the BLER (i.e. outage) and the throughput. Furthermore, we can confirm the tendency that the throughput is enhanced more substantially for long distances $d_{\mathrm{S}}$, when there is a larger number of relay candidates $N_{\mathrm{R}}$.

\section{CONCLUSION}

In this paper, an EXIT chart aided relay activation scheme was proposed for JCN coded systems. The main problem to be solved was to formulate the decision criteria, whether to activate "two-hop transmission" or "XOR assisted transmission" in the $\mathrm{R}$ nodes of the network. In order to address the problem, the criteria were related to the predicted EXIT trajectories of all potential $\mathrm{R}$ nodes. We demonstrated that the EXIT chart aided relay activation scheme is effective in terms of improving both the outage probability of the communication link, as well as the throughput of the network.

\section{REFERENCES}

[1] A. Sendonaris, E. Erkip, and B. Aazhang, "User cooperation diversitypart I: System description,” IEEE Trans. Commun., vol. 51, no. 11, pp. 1927-1938, Nov. 2003.

[2] A. Nosratinia, T. E. Hunter, and A. Hedayat, "Cooperative communication in wireless networks," IEEE Commun. Mag., vol. 42, no. 10, pp. 68-73, Oct. 2004.

[3] R. Ahlswede, N. Cai, S.-Y. R. Li, and R. W. Yeung, "Network information flow," IEEE Trans. Inform. Theory, vol. 46, no. 4, pp. 1024-1216, July 2000.

[4] Y. D. Chen, S. Kishore, and J. Li, "Wireless diversity through network coding," in Proc. WCNC '06, vol. 3, Las Vegas, NV, USA, Apr. 2006 , pp. 1681-1686.

[5] L. Xiao, T. E. Fuja, J. Kliewer, and J. Daniel J. Costello, "A network coding approach to cooperative diversity," IEEE Trans. Inform. Theory, vol. 53, no. 10, pp. 3714-3722, Oct. 2007.

[6] C. Hausl and J. Hagenauer, "Iterative network and channel decoding for the two-way relay channel," in Proc. ICC'06, Istanbul, Turkey, June 2006, pp. 1568-1573.

[7] C. Hausl and P.Dupraz, "Joint network-channel coding for the multipleaccess relay channel," in Proc. SECON'06, Reston, VA, USA, Sept. 2006, pp. 817-822.

[8] S. ten Brink, "Convergence behavior of iteratively decoded parallel concatenated codes,", IEEE Trans. Commun., vol. 49, no. 10, pp. 17271737, Oct. 2001.

[9] S. ten Brink, G. Kramer, and A. Ashikhmin, "Design of low-density parity-check codes for modulation and detection," IEEE Trans. Commun., vol. 52, no. 4, pp. 670-678, Apr. 2004.

[10] A. Goldsmith, Wireless Communication. New York; Cambridge Press, 2005.

[11] K. Yamamoto and S. Yoshida, "Tradeoff between area spectral efficiency and end-to-end throughput in rate-adaptive multihop radio networks,' IEICE Trans. Commun., vol. E88-B, no. 9, pp. 3532-3540, Sept. 2005.

[12] J. Hagenauer, "The turbo principle in mobile communications," in Proc. ISITA, XI'AN, Peoples Republic of China, Oct. 2002.

[13] F. Brannstrom, "Convergence analysis and design of multiple concatenated codes," Ph.D. dissertation, Chalmers University of Technology, 2004.

[14] J. Hagenauer, E. Offer, and L. Papke, "Iterative decoding of binary block and convolutional codes," IEEE Trans. Inform. Theory, vol. 42, no. 2, pp. 429-445, Mar. 1996. 